\title{
La politique des transports en Tchécoslovaquie dans l'entre-deux-guerres :à la recherche d'un nouvel équilibre dans un contexte de concurrence intermodale
}

The long march toward a multimodal management of public transportation in the Interwar period Czechoslovakia

Jan Oliva

\section{CpenEdition}

Édition électronique

URL : https://journals.openedition.org/rhcf/1522

DOI : $10.4000 /$ rhcf. 1522

Éditeur

Rails \& histoire

Édition imprimée

Date de publication : 1 septembre 2012

Pagination : 27-47

ISSN : 0996-9403

Référence électronique

Jan Oliva, «La politique des transports en Tchécoslovaquie dans l'entre-deux-guerres à la recherche d'un nouvel équilibre dans un contexte de concurrence intermodale », Revue d'histoire des chemins de fer [En ligne], 42-43 | 2012, mis en ligne le 14 novembre 2014, consulté le 22 avril 2022. URL : http:// journals.openedition.org/rhcf/1522 ; DOI : https://doi.org/10.4000/rhcf.1522 
Jan OLIVA

\section{La politique des transports en Tchécoslovaquie dans l'entre-deux-guerres : à la recherche d'un nouvel équilibre dans un contexte de concurrence intermodale}

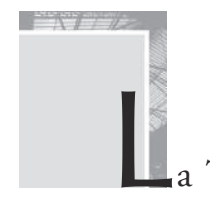

Tchécoslovaquie fut fondée le 28 octobre 1918 sur les ruines de l'empire austro-hongrois. La partie occidentale du nouvel État (en particulier la Bohème centrale, l'arc frontalier septentrional, la Moravie du Nord) possédait une industrie développée qui avait été traditionnellement exportatrice de produits manufacturés au sein du " marché commun » de la double Monarchie. À l'issue de la Grande Guerre, elle se retrouva brutalement privée de ses débouchés et dans une situation économique critique. Au temps de l'Autriche-Hongrie, ses usines approvisionnaient les vastes régions rurales de la Monarchie et ses deux capitales, Vienne et Budapest, en utilisant le réseau ferré adapté en conséquence. L'économie austro-hongroise fonctionnait dans une large mesure en vase clos. En ce qui concerne les échanges en provenance de l'outre-mer, la Monarchie disposait de ports maritimes sur la côte adriatique (dont le plus important fut Trieste), lui assurant un trafic de marchandises sans l'intermédiaire de pays étrangers. Cette donne changea à l'issue de la Grande Guerre. La Tchécoslovaquie devint un pays intérieur, très éloigné des ports maritimes (la distance séparant Prague de Trieste était d'environ $830 \mathrm{~km}$, de Stettin d'environ $530 \mathrm{~km}$, de Hambourg d'environ $700 \mathrm{~km}$ ). Ce handicap fut par la suite atténué par les traités de paix. 
Cependant, la situation géographique centrale en Europe et la forme longitudinale du pays représentaient également un avantage stratégique. Les hommes politiques et les économistes comptaient sur le fait que leur pays serait traversé par d'importants flux de transit du commerce international. Ils espéraient pouvoir conserver deux anciens couloirs de transit austro-hongrois : l'axe Berlin-Prague-Vienne et l'axe Galicie-Ostrava-Brno-Vienne qui correspondaient respectivement à la liaison la plus courte entre Berlin et Vienne, les deux anciennes capitales d'empires " amis ", et au vieil axe ferroviaire ayant relié les régions industrielles et minières les plus anciennes de la Transleithanie à leur capitale.

Prague, la capitale tchécoslovaque, par sa situation géographique et la volonté politique des Alliés, convoitait le rôle d'important nœud de transport en Europe centrale. Du point de vue du trafic international, ce nœud devait être ferroviaire, routier et aéronautique. La ville devait se hisser au même niveau que Vienne, Budapest ou Varsovie. Parallèlement, des nœuds secondaires jusqu'alors quasiment inexistants étaient à construire afin de compléter le schéma national des transports, conçu encore dans une perspective monomodale.

La période de l'entre-deux-guerres fut aussi celle de l'émergence de l'aviation et de la maturation rapide du transport routier. Ces nouveaux acteurs reçurent un accueil inégal de la part des pouvoirs publics. Nous tentons ici de dessiner les grandes lignes de la politique tchécoslovaque en matière de transports en montrant tout d'abord combien il était difficile pour le nouvel État de se détacher des schémas de fonctionnement austro-hongrois. Nous esquissons ensuite l'évolution des différents modes de transport, qui ne fut pas dépourvue de tensions. Enfin, nous démontrons que la réforme institutionnelle réalisée en 1938 fut davantage liée aux circonstances politicoéconomiques qu’à une réelle volonté du gouvernement.

\section{Un mode de gestion des transports hérité de la période austro- hongroise}

\section{La répartition des compétences en matière de gestion des transports}

Dans les premiers moments qui suivirent la fondation de l'État tchécoslovaque à la fin d'octobre 1918, la priorité des responsables politiques était d'assurer à tout prix la stabilité intérieure du pays, le calme et la continuité de la vie quotidienne. Dans le domaine des transports, cet effort revenait à faire 
fonctionner les réseaux des chemins de fer sans encombre. Dans un premier temps, l'ensemble du corpus législatif de la période austro-hongroise fut repris et sa validité prolongée jusqu'à ce que la nouvelle République fût en mesure de le remplacer par ses propres lois, plus adaptées à ses besoins et à son époque.

En parallèle avec la législation, la structure administrative fut également transposée, ce qui, dans le domaine des transports, signifiait, d'une part, la création d'un portefeuille ministériel dédié aux chemins de fer et, d'autre part, la répartition des autres responsabilités entre d'autres ministères. En définitive, cinq ministères différents furent concernés :

- ministère des Chemins de fer (Ministerstvo železnic) pour le transport ferroviaire, mais aussi, à partir de 1928, pour le transport routier public (autobus et camions) ;

- ministère des Postes et des Télécommunications (Ministerstvo Pośt a telekomunikaci) pour le transport sur route (autobus et camions) jusqu'en 1932, l'année d'incorporation de ces services au ministère des Chemins de fer ;

- ministère des Travaux publics (Ministerstvo Verejných praci) pour le transport aérien et pour les réalisations d'infrastructures en général (routes, chaussées, électrification...) ;

- ministère du Commerce et de l'Industrie (Ministerstvo Obchodu a živnostî) pour ses activités au niveau du transport des marchandises, import et export et pour le transport fluvial ;

- ministère de l'Intérieur (Ministerstvo vnitra) pour la police.

Nous devons ajouter à ce propos les interventions régulières du ministère de la Défense du fait du caractère stratégique des infrastructures ${ }^{1}$ et à cause des " services " gratuits dont le rail en particulier était chargé pour assurer le transport du matériel militaire et des troupes.

Dès 1918, cette organisation "à l'autrichienne " des transports publics avait paru désuète à certains. Par conséquent, la période de l'entre-deuxguerres fut ponctuée de diverses tentatives de réforme, plus ou moins réussies, selon le contexte politique et économique général.

La première tentative de réforme précéda même la création de la République, car les auteurs du premier programme économique du pays avaient l'intention de créer une administration commune à l'ensemble des transports au sein de laquelle une politique de transport globale aurait été

1- Un exemple : le 17 septembre 1928 le gouvernement imposa au ministère des Chemins de fer d'élaborer en commun avec le ministère de la Défense un "programme d'investissements élargi » pour répondre aux besoins en matière de défense du territoire (" programme militaire d’investissement ferroviaire ", železniční vojenské investični program). 
élaborée 2 . Dans notre analyse a posteriori, cette tentative, louable et sûrement visionnaire, ne pouvait aboutir, en 1918 du moins, du fait de la différence de maturité entre les modes de transports, du poids de l'héritage austro-hongrois et de l'urgence économique. En effet, fort de son histoire déjà presque séculaire et de son organisation matérielle et humaine considérable, «le » chemin de fer dominait totalement la sphère des transports par rapport aux débuts encore timides des transports routier et aérien. Les rapports de force étaient cependant appelés à évoluer considérablement au cours de l'entre-deux-guerres.

\section{Le partage des transports entre le secteur privé et le secteur public} et l'instauration de fait d'un monopole de transport public

La tendance générale en Tchécoslovaquie de l'après-guerre allait à l'instauration de grands monopoles publics auxquels ne pouvait échapper le secteur des transports. Cela concernait en premier lieu les chemins de fer à cause, bien entendu, de leur importance économique et stratégique, mais aussi de leur poids social. En effet, avec quelques 150000 employés et un budget annuel dépassant le milliard de couronnes tchécoslovaques, les Chemins de fer tchécoslovaques d'État (Československé státni dráhy, ČSD) constituaient la première entreprise nationale.

Depuis le début du $\mathrm{xx}^{\mathrm{e}}$ siècle, l'Autriche poursuivait une nationalisation systématique des grandes compagnies ferroviaires. Le nouvel État tchécoslovaque prolongea cette politique en exprimant, dès ses débuts, le principe de la propriété publique des chemins de fer. Étant donné que, en 1918, le transport sur rail représentait en Tchécoslovaquie encore la quasi-totalité du transport terrestre à moyenne et longue distance, on peut dire que l'État avait l'intention de s'assurer une position de monopole dans le domaine des transports.

$\mathrm{Si}$, en revanche, le transport sur route demeurait dans un premier temps " délaissé " au profit du secteur privé, puis seulement timidement et progressivement partagé entre le privé et le public, c'est que l'utilisation du véhicule à moteur n'était pas encore suffisamment répandue et intégrée au mode de vie des Tchécoslovaques. En effet, les progrès majeurs dans cette technologie datent seulement des années de guerre. Le territoire de la future ČSR resta alors quasiment à l'écart de l'usage de l'automobile à des fins militaires à cause de sa situation géographique éloignée des zones de combats. Après la guerre, le gouvernement ne s'intéressa que timidement aux possibilités offertes par le véhicule à moteur via le ministère des Postes et des Télécommunications

2- Cf. František Hodáč, «O náš hospodářský program » [Pour notre programme économique], Obzor národohospodárskeý, Ročník XXIII, 1926, p. 1-43. 
chargé de les tester ${ }^{3}$. En attendant d'obtenir des résultats fiables et étayés, le recours au véhicule à moteur comme mode de transport de voyageurs demeura essentiellement le fait de l'initiative et du profit du secteur privé. En effet, l'euphorie des premiers mois de l'après-guerre faisait croire en la réalisation d'un vaste plan ferroviaire d'investissement qui aurait comblé les lacunes du réseau pour lesquelles la mauvaise volonté de Vienne au temps de l'Empire austrohongrois fut le coupable désigné. De fait, la politique publique en matière de transport allait se concentrer dans un premier temps presqu'exclusivement sur le rail. Ce ne fut que bien plus tard, devant l'impossibilité d'honorer le nombre grandissant de demandes de construction de lignes de chemin de fer nouvelles d'une part, et sous la pression des entrepreneurs particuliers soumissionnant pour des concessions de lignes de bus d'autre part, que l'État, par l'intermédiaire de ses deux ministères, celui des Postes et Télécommunications et celui des Chemins de fer, commença à devenir plus actif dans le domaine du transport routier. Son action s'inscrivait alors dans un double registre : créer un cadre législatif et fiscal encadrant minutieusement le développement de l'entrepreneuriat automobile privé, protéger ses propres entreprises de transport de cette concurrence et les renforcer.

Dans le domaine du transport aérien, la ČSR poursuivit tout au long de la période étudiée une politique cohérente de monopole intérieur d'une part, incarné par la compagnie des Lignes aériennes tchécoslovaques d'État (ČSA, Československé státni aerolinie), et de prise de participation dans le réseau aérien international par le biais des parts détenues dans le capital de la Compagnie aérienne tchécoslovaque (ČLS, Československá letecká společnost), d'autre part. Cette politique fut étayée par la construction d'un réseau d'infrastructures au sol, d'aéroports bien entendu, mais aussi de réseaux techniques connexes - phares et signalétiques pour la navigation diurne et nocturne, stations météorologiques, téléphonie, etc. Le talon d'Achille de la politique en faveur de l'aviation était constitué par l'enveloppe financière qui lui était dédiée. Suffisante et même généreuse au début des années 1920, cette enveloppe ne suivit pas l'évolution technologique et les exigences nouvelles en matière d'équipements au sol en particulier. Aussi le ministère des Travaux publics, chargé du portefeuille de l'aviation, de la conception et de la réalisation des travaux, dut-il se résigner à concentrer ses efforts sur quelques sites aéroportuaires choisis, en premier lieu sur Prague. Ainsi la capitale tchécoslovaque put devenir un nœud aérien européen et bénéficier

3- La première ligne de bus ouverte à nouveau après l'armistice fut celle de Pardubice à Bohdaneč, dès l'automne 1918. Il s'agit en l'occurrence de la première ligne d'autobus des Postes en Autriche-Hongrie, datant de 1906. 
d'une renommée internationale bien méritée grâce notamment à la qualité de ses aéroports, Prague/Kbely d'abord, puis Prague/Ruzyně ouvert au trafic en 1937.

En 1918, les transports tchécoslovaques reprirent donc les grandes lignes organisationnelles et réglementaires de "l'empire défunt " ${ }^{4}$. Cette organisation, si elle se justifiait alors du point de vue économique et technologique, allait pourtant avoir des conséquences inégales et controversées sur le développement des différents modes de transport au cours des vingt années suivantes. Elle constituait certes un puissant levier pour les uns, mais elle freina le développement des autres.

\section{Les transports durant l'entre-deux-guerres : des évolutions inégales et controversées}

\section{Les chemins de fer, expression de la politique de la cohésion nationale}

Le nouvel État ne pouvait envisager un autre avenir pour son réseau ferré que celui de la propriété publique. Aussi, dès les premières séances du Conseil révolutionnaire, la nationalisation du réseau fut-elle décidée. Cette politique n'était pas une nouveauté. Elle s'inscrivait dans la logique des nationalisations des grandes compagnies entamée de façon « définitive " par l'État autrichien dès 1906. La Tchécoslovaquie hérita par conséquent d'un réseau ferré en grande partie nationalisé (environ $11400 \mathrm{~km}$ sur les quelque 13500 situés sur son territoire en 1918) et put fonder comme on l'a dit sa compagnie nationale ČSD dès 1919. Afin de compléter le réseau d'État, il fut décidé de nationaliser progressivement les fragments manquants ; ce sont le contexte économique et la pénurie financière qui obligèrent les gouvernements successifs à étaler le processus dans la durée.

Après s'être assuré la loyauté et la coopération de la compagnie $\mathrm{KBD}^{5}$ qui assurait la seule véritable connexion importante avec la Slovaquie et possédait de ce fait une importance stratégique et économique cruciale, le gouvernement décida la nationalisation des deux autres grandes compagnies privées situées dans des bassins miniers et industriels de la Bohême septentrionale $\left(1^{\text {er }}\right.$ janvier 1923 rachat de $\mathrm{UTD}^{6}$; 15 juin 1923 rachat de la compagnie de Buštěhrad). Il fut décidé dans un second temps d'incorporer des lignes d'importance

4- FETJÖ, 1993.

5- KBD : Košicko-Bobuminská dráha, Compagnie de chemin de fer de Košice à Bohumín.

6- UTD : Ústecko-Teplická drába, Compagnie de chemin de fer de Ústí à Teplice. 
moindre, souvent déjà exploitées par l'État, et dont le bilan financier était, pour la plupart d'entre elles, déficitaire?

Mais si la volonté de nationalisation était le support et la ligne directrice de la politique ferroviaire nationale, elle était également fondée sur un autre enjeu, essentiel. Le réseau tchécoslovaque présentait en effet des disparités et des déséquilibres, en particulier en ce qui concerne les connexions transversales ouest-est, dont les effets devaient être compensés par une politique de construction ambitieuse. Entre les deux parties de la République - les pays tchèques, à l'Ouest, d'une part, et la Slovaquie avec la Russie subcarpatique, à l'Est, d'autre part, l'activité de construction ferroviaire prit logiquement deux visages différents. En effet, les pays tchèques disposant déjà d'un réseau ferré développé ne pouvaient prétendre, dans la situation de crise de transport que connaissait l'après-guerre, à des réalisations de lignes nouvelles. Les travaux se concentrèrent par conséquent sur des chantiers de petite et de moyenne taille, essentiellement de rénovation des infrastructures ${ }^{8}$, de remodelage des nœuds ferroviaires importants ${ }^{9}$, d'élargissement des itinéraires qui étaient récemment devenus des axes de trafic lourd, de (ré)aménagement des grands centres urbains, en premier lieu de Prague, mais aussi de $B_{r n o}{ }^{10}$ et Bratislava, de raccordements transfrontaliers, en particulier vers la Slovaquie ${ }^{11}$, et de travaux d'équipements divers.

La Slovaquie, en revanche, devait bénéficier d'un effort de construction de lignes nouvelles, car son réseau ferré, lacunaire, possédait bien plus encore que le réseau des pays tchèques le défaut d'être happé en quelque sorte par "son " ancienne capitale, Budapest. Il fallait dans ce contexte créer rapidement un axe transversal de direction ouest-est, qu'on allait nommer "l'axe magistral slovaque " (slovenská magistrála). La mise en œuvre de ces projets

7- La loi no 156/1925 du 30 mars 1925 permit une première vague de nationalisations de quelques 48 compagnies réunissant une longueur totale de lignes de $1589 \mathrm{~km}$. Votée en même temps, la loi n $157 / 1925$ permit l'achat massif d'actions de la compagnie KBD, mentionnée supra. Dans son cas il s'agit d'une nationalisation progressive par le biais de la participation au capital, la compagnie restant cependant privée de iure jusqu'en 1948.

8- On réalisa par exemple immédiatement après la prise du pouvoir en novembre 1918 la reconstruction de la ligne locale Břeclava - Kúty - Děvínská Nová Ves pour améliorer la connexion des capitales Prague et Bratislava.

9- On rénova par exemple entièrement le carrefour ferroviaire de Břeclav devenu international du fait de sa proximité avec la nouvelle frontière avec l'Autriche.

10- Construction du raccordement urbain «komárovská spojka » en 1927.

11- Construction ou rénovation de lignes Vsetín - Bylnice - Brumov, Veselí nad Moravou Nové Mesto nad Váhom et Horní Lideč - Púchov nad Váhom. 
exprimait à la fois matériellement et symboliquement, dans le domaine des transports, la politique de cohésion nationale, un leitmotiv politique récurrent. Cette politique trouva sa première consécration dès 1920, après deux années à peine d'études et de sondages, dans le vote d'une loi fondamentale sur la construction de lignes nouvelles ( $\$ 1920 / 235)$, puis se prolongea par une longue période de travaux qui, dépassant largement le cadre initialement prévu, s'étendit sur l'ensemble de la période de l'entre-deux-guerres ${ }^{12}$ (fig. 1).

L'ensemble du réseau ferré tchécoslovaque, dont nous avons constaté le déséquilibre existant entre ses parties ouest et est, trouva grâce à cette double politique de nationalisation et de construction une nouvelle cohérence. Celleci fut renforcée par la gestion rigoureusement centralisée et hiérarchisée incarnée par le ministère des Chemins de fer ${ }^{13}$.

\section{Le transport routier : un casse-tête politique et financier ${ }^{14}$}

\section{La politique ambiguë de l'État vis-à-vis du transport routier}

Le transport routier avait sur le mode ferroviaire plusieurs avantages qui ne cessèrent de se renforcer grâce au perfectionnement technique des véhicules à moteur et, conjointement, des infrastructures routières. En Tchécoslovaquie, les hauts fonctionnaires étaient conscients dès le début des années 1920 des atouts dont disposait le transport routier (plus rapide sur petite et moyenne distance, meilleur marché pour des petites quantités, s'adaptant rapidement à la demande, plus flexible). Ils savaient aussi qu'il allait se développer, même s'ils admettaient la difficulté de mesurer au juste de quelle façon. L'erreur fondamentale se produisit au moment où ces fonctionnaires, pour la plupart employés du ministère des Chemins de fer, de concert avec les hommes politiques soucieux de protéger le monopole d'État et la société professionnelle cheminote, définirent le transport routier comme concurrent indésirable, voire comme un danger potentiel pour l'avenir du transport ferroviaire. Le fait de penser et d'agir à ce point en termes de concurrence biaisa par la suite la politique des transports de l'État.

12- Ses différents jalons étaient, dans l'ordre chronologique, les lignes : Bánovce nad Ondavou - Vajany (mise en service en octobre 1921), Zvolen - Krupina (janvier 1925), Handlová - Horná Štubňa (décembre 1931) et la ligne la plus longue Červená Skála - Margecany, 92 km (mise en service en juillet 1936).

13- En effet, en dehors des investissements liés à la nature particulière de chacune des composantes géopolitiques de l'État, le ministère s'efforça, en tenant compte de la situation sur le terrain, de doter l'ensemble du pays d'équipements appropriés - ateliers d'entretien et de réparation du matériel roulant, structures d'hébergement pour les cheminots, etc.

14- Le premier à avoir traité de la problématique du développement du transport routier dans la ČSR de l'entre-deux-guerres fut ŠTEMBERK, 2004. 


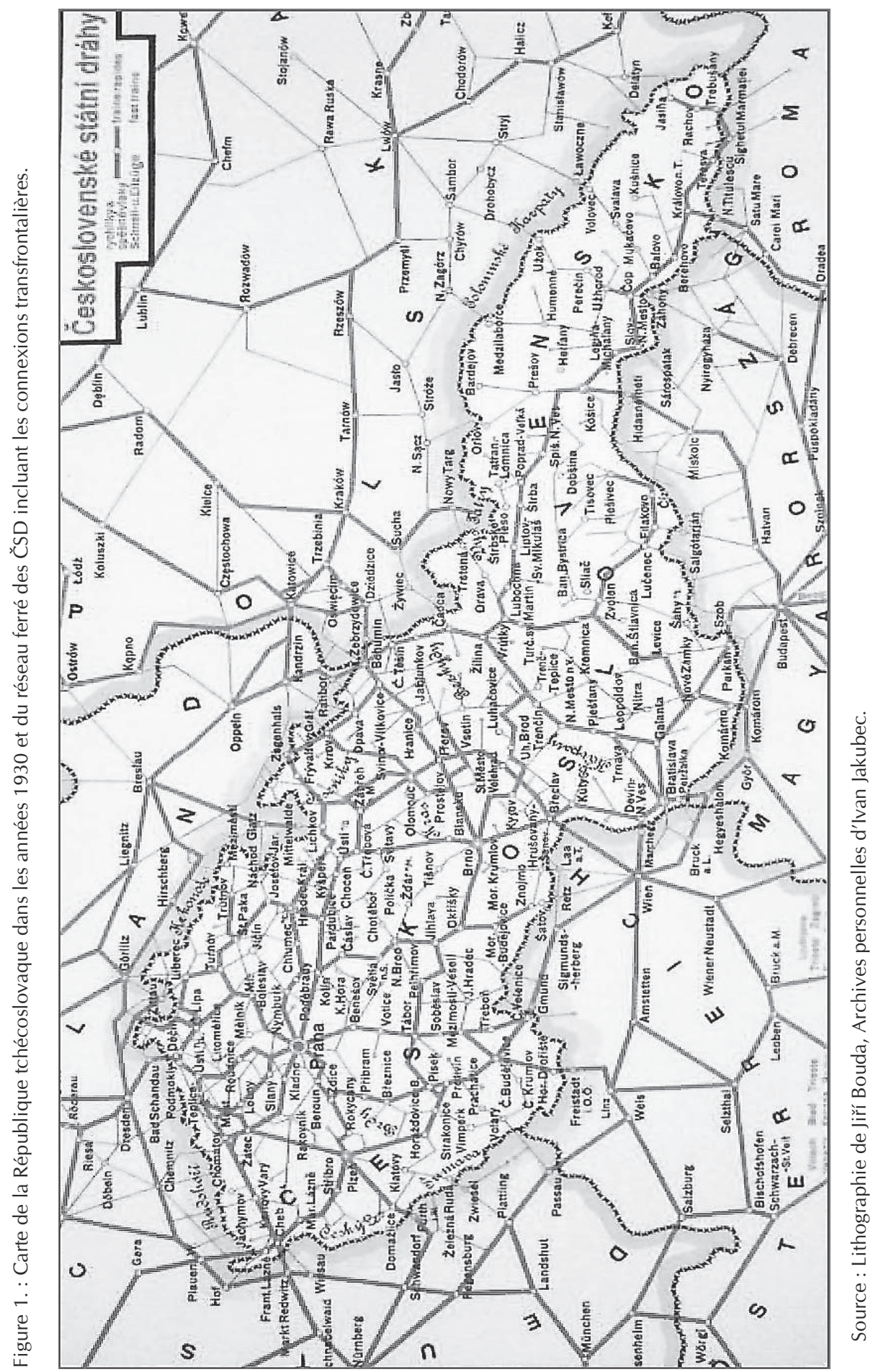


Le monopole de transport exercé par les chemins de fer doublé d'un encadrement étroit du secteur routier privé faisait craindre, ou espérer selon les interprétations, son extension également à ce domaine de l'économie en plein essor. En effet, depuis la création de la République, deux entreprises publiques étaient actives dans le domaine routier : d'abord le ministère des PTT pour son compte, comme nous l'avons évoqué supra et, depuis 1927 environ, le ministère des Chemins de fer par le biais de l'entreprise ČSD au sein de laquelle une direction du transport routier fut créée. Le fait que le ministère des Chemins de fer avait investi dans le transport routier fut un signe évident de la montée en puissance de ce nouveau mode de transport. Le ministère souhaitait étudier son potentiel, lutter contre la concurrence privée et créer à terme une alternative à la construction de nouvelles voies ferrées. L'objectif final était clairement le maintien de son monopole historique de transport.

Les premières tentatives de création de lignes d'autocar publiques s'avérèrent peu efficaces dans ce domaine encore très concurrentiel. Arguant du fait que les transporteurs routiers exerçaient leur activité dans des conditions trop avantageuses pour eux (ne payant pas les routes, ni les taxes de transport), le lobby ferroviaire et, en premier lieu, le ministère des Chemins de fer obtinrent du gouvernement et du Parlement le vote d'une série de lois entre 1927 et $1935^{15}$ modifiant totalement le rapport des forces à l'avantage du rail (fig. 2). De plus, le ministère obtint en 1932 l'incorporation en son sein de tous les services de transport routier public, augmentant d'un seul coup et de manière significative la taille de son département routier et son poids économique. À partir de cette date, le ministère des PTT ne fut plus que commanditaire de services routiers auprès du ministère des Chemins de fer. Cette position nouvelle, l'État tchécoslovaque ne l'abandonna plus durant la période étudiée, au mieux, il tenta de réorganiser le secteur des transports afin d'en améliorer la rentabilité. Sa politique, dirigée ouvertement contre le secteur routier privé dont la caractéristique principale était son extrême fractionnement ${ }^{16}$, rencontra naturellement une vive opposition qui trouva son paroxysme en 1932.

15- Il s'agissait principalement des lois suivantes : sur le Fonds routier (loi n 116/1927 du 14 juillet 1927 modifiée en 1931) qui réorganisa la législation fiscale des véhicules à moteur et introduisit une taxe pour le transport par autocar (30\% du prix du billet, hors le transport à l'intérieur des agglomérations), sur la taxation des huiles minérales (loi n $\left.{ }^{\circ} 77 / 1931\right)$, sur la mobilisation des véhicules à moteur pour les besoins de l'armée (loi nº 68/1932 du 12 mai 1932) et sur le transport par véhicules à moteur (loi n 198/1932 du 23 décembre 1932, modifiée en 1935).

16- En effet, plus de $50 \%$ des entreprises étaient composées d'une seule personne ; le nombre moyen de véhicules par entreprise était de 1,3. Voir Ing. Dr. Antonín Patočka, Koncesovaná doprava nákladi motorovými vozidly [Le transport concédé de marchandises par véhicules à moteur], Prague, 1948, p. 1-19. 
En janvier de cette année-là, épuisés par ce qu'ils vivaient comme d'incessantes persécutions à l'encontre de leur métier, les transporteurs privés, dont il faut pourtant souligner le caractère individualiste, appelèrent à une journée de manifestation nationale dans les grandes villes (en particulier à Prague) suivie d'une journée de grève générale pour protester contre l'amendement de la loi sur le Fonds routier. Cette opposition n'eut cependant pas d'effet immédiat car, la même année et à nouveau trois ans plus tard, une loi encore plus contraignante à leur égard fut votée ${ }^{17}$.

Figure 2. Les effets des lois de 1932 et 1935 sur l'évolution du parc tchécoslovaque d'autocars.

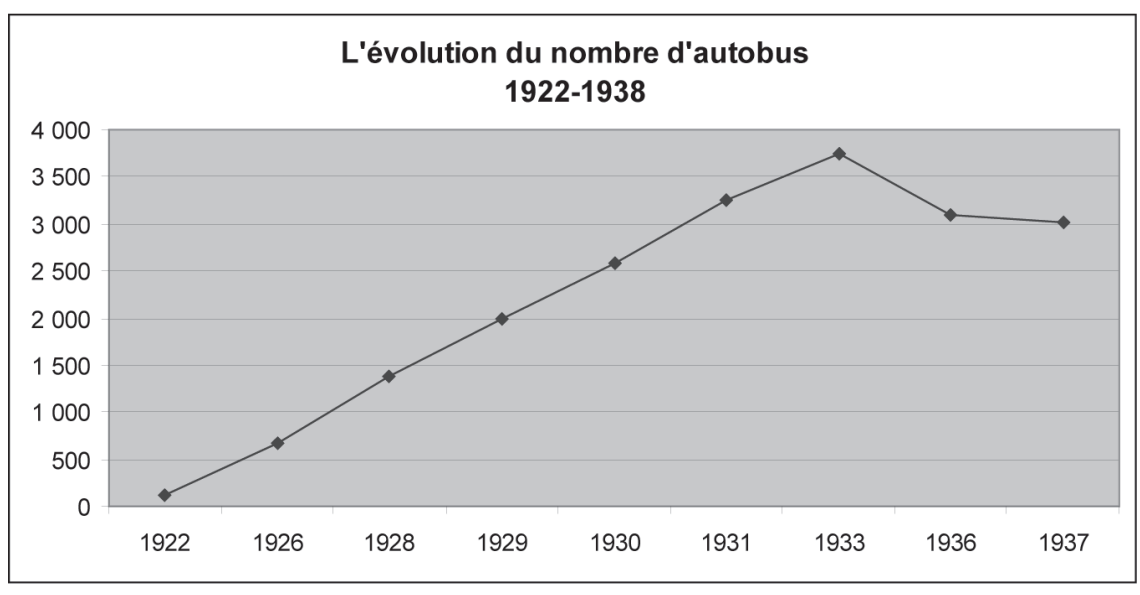

Source: Graphique de l'auteur, à partir des données de la Statistická př́ručka rep. Československé, volume V. 1934 (p. 284) et IX. 1938 (p. 118).

\section{Le fardeau financier représenté par les infrastructures routières}

Similaires, par leur nature, aux lignes des chemins de fer, les routes semblaient prédisposées à être à leur tour "nationalisées ", dans le sens de leur gestion exclusive par l'État. En effet, la gestion routière était partagée entre les collectivités territoriales, les communes, les " cercles " (équivalent des départements français), les " pays " (Bohême, Moravie, Slovaquie, Russie subcarpatique) et, enfin, l'État par l'intermédiaire de son ministère des Travaux publics. Nous avons vu cependant que l'État affichait comme sa priorité première la reconstruction du réseau ferré. Le décalage entre les bonnes intentions et la réalité, déjà parfois visible dans l'agenda du ministère des Chemins de fer, fut encore bien plus important en ce qui concerne la gestion du réseau routier.

17- Cf. supra note 14. 
On pouvait lire dans l'article " La nationalisation des routes " paru en mai $1922^{18}$ : "La nationalisation des routes d'importance nationale est une affaire évidente. " Mais, pour financer l'opération, il fallait disposer d'un budget conséquent. En effet, en 1922, $5871 \mathrm{~km}$ de routes appartenaient déjà à l'État, ce qui pesait dans son budget pour un montant de 26 millions de couronnes. Le nouveau plan portait sur $4122 \mathrm{~km}$ de routes nouvelles, pour les réparations et l'entretien desquelles il aurait fallu mobiliser 200 à 300 millions de couronnes supplémentaires. L'article dressait ensuite la liste de tous les autres grands projets d'investissements publics, à savoir la construction ferroviaire (qui disposait pour la réalisation du plan de construction de 1922 d'un budget astronomique de 6,5 milliards), l'électrification du pays, un plan d'urgence qui réservait 12 millions annuellement à des régions à fort taux de chômage, etc. Cet article montre que l'État avait bien l'intention d'agir sur de nombreux fronts, afin de développer, tout en les contrôlant, toutes les infrastructures et réseaux d'envergure nationale, non seulement ceux des transports, mais aussi d'énergie. Mais les moyens disponibles nécessitaient une hiérarchisation des tâches et les infrastructures routières partageaient alors la seconde place avec les aéroports et les aménagements des voies d'eau. La publication jubilaire parue en 1928, intitulée "Dix ans de la République tchécoslovaque ${ }^{19}$, qui dressait a priori un tableau exclusivement positif des efforts de construction de l'État, dut, cependant, reconnaître que le manque d'entretien des routes durant la Grande Guerre, mais aussi les « faibles moyens financiers » consentis à la construction routière conjugués avec la hausse du trafic, avaient conduit à une détérioration générale du réseau routier national : «L'effort des dernières années, dont l'augmentation progressive du budget consacré à la construction routière est le reflet, n'a pas pu sauver de la destruction l'ensemble de notre réseau routier à la fin de l'année 1927. " Ce triste constat devait servir de déclencheur pour la réorganisation du financement de la construction routière, à commencer par la création d'un Fonds routier d'État ${ }^{20}$ en 1927. Ce fonds était alimenté par les recettes de taxes et des douanes perçues par l'État sur les produits à destination de l'industrie automobile (produits pétroliers, pneus, caoutchouc, etc.). Grâce à ce système, la remise en état du réseau fut réelle, mais elle ne concerna, dans un premier temps, que les axes principaux. C'est pourquoi encore en 1932 on pouvait lire dans la presse un article détaillé

18- "Sestátňovacísilniční akce » [L’action de nationalisation des routes], Právo Lidu, 18 mai 1922, Archives du ministère des Affaires étrangères, MZV-VA, carton 1864.

19- Deset let republiky československé, tome II, Prague, 1928.

20- En tchèque : Státní silnični fond, cf. note 14. 
sur un plan décennal exigeant l'accélération de la construction routière dans les pays tchèques, pourtant les mieux lotis sur le plan national, et la prise en charge partielle de cette tâche par les autorités territoriales ${ }^{21}$.

Cependant, comme nous l'avons exposé précédemment, le renouveau de la construction routière se fit aux frais des usagers de la route et de l'industrie automobile, ralentissant d'autant son expansion et enfermant le transport automobile tchécoslovaque dans un vase clos au potentiel de développement limité.

\section{La voie d'eau : un auxiliaire des chemins de fer}

À la différence du transport routier, la voie d'eau obtint dès l'aprèsguerre un statut spécifique et protégé dû autant à des considérations intérieures qu'extérieures.

Sur le plan extérieur d'abord, la délégation tchécoslovaque envoyée en France pour débattre des clauses des Traités de paix avait pour mission d'obtenir "l'internationalisation " des voies de communication, parmi lesquelles figurèrent au premier plan les cours d'eau Labe (Elbe), Danube et Odra ${ }^{22}$. En ajoutant cette extension internationale à son réseau de transport intérieur, la République poursuivait un double objectif, économique et stratégique. On souhaitait notamment rendre le pays plus indépendant des contraintes tarifaires imposés pour le transit de ses biens et marchandises et ménager des connexions qui constitueraient une alternative en cas d'un conflit armé avec l'un de ses voisins.

Sur le plan intérieur, la raison pour laquelle la voie d'eau jouissait d'un statut privilégié était également double. Premièrement, la politique en sa faveur entraîna la création de compagnies de navigation "tchécoslovaques " $^{23}$ (une pour chacun des cours d'eau mentionnés) avec la participation financière de l'État qui donnait des garanties politiques et financières au développement dans l'avenir de ce mode de transport. L'État s'engagea notamment - à l'image des garanties accordées autrefois aux concessionnaires ferroviaires - à rémunérer au minimum le capital investi, à effectuer certains travaux d'aménagement

21- «V deseti letech mají býti všechny okresní silnice v Čechách postaveny a uvedeny do pořádku » [En Bohême, toutes les routes des cercles doivent être construites et rénovées dans dix ans], Doprava, janvier 1932.

22- Le principe de l'internationalisation des cours d'eau fut inscrit dans l'art. 331 du Traité de Versailles et exécuté dans des «Actes de navigation » : Labská plavební akta, signé à Dresde le 22 février 1922 et complété par un protocole signé à Paris le 31 mars 1923; Úmluva stanovicí definitivní Dunajský Statut signé à Paris le 23 juillet 1921 ; celui concernant l'Odra n’a pas été réalisé. 23- La loi n 188/1922 régulait la navigation sur l'Elbe et le Danube, la loi nº 64/1924 celle de l'Odra. 
(écluses ; barrages ; ports), à investir dans la modernisation de la flotte ${ }^{24}$ et, indirectement, à harmoniser sa politique de transport entre le rail et la voie d'eau.

D’une part les responsables politiques tchécoslovaques étaient parfaitement conscients de l'importance de la voie fluviale dans les relations avec l'étranger éloigné, via, notamment, les ports marins, situés à l'embouchure de l'Elbe pour la mer du Nord et du Danube pour la mer Noire, d'autre part ils imaginaient que les pays voisins, avec lesquels les relations s'annonçaient tendues, allaient avoir tendance à dresser des barrières tarifaires sur les chemins de fer, afin d'entraver la marche économique du pays. Finalement, ce fut l'inverse qui se produisit. Les tarifs des compagnies ferroviaires allemandes et polonaises notamment furent si bas que l'utilisation de la voie navigable demeurait, si l'on calculait les dépenses totales pour son aménagement, son entretien et son usage, toujours tributaire des subventions étatiques.

Deuxièmement, et c'est sans doute l'élément essentiel, la voie d'eau ne concurrençait pas, physiquement, le réseau ferré. En effet, les deux seuls tronçons ouverts à la navigation à vapeur pendant toute l'année se situaient en périphérie du pays - le Danube longeait la frontière d'avec l'Autriche et la Hongrie sur $172 \mathrm{~km}$ et l'Elbe desservait uniquement sur quelques $200 \mathrm{~km}$ le Nord de la Bohême en frôlant son centre ${ }^{25}$. Aussi l'essentiel du trafic sur ces fleuves servait-il aux échanges avec l'étranger (pour la période 1932-1936 dans une moyenne de $91,2 \%$ pour le Danube et de $64,7 \%$ pour l'Elbe) et non pas comme support logistique du marché intérieur (respectivement 8,8 et $35,3 \%$ ). Ces deux cours d'eau - peut-on parler de réseau fluvial ? - pouvaient donc être pensés comme des prolongements à l'étranger du réseau ferré national dans le but de conserver la totalité des bénéfices dans le giron des compagnies tchécoslovaques, mais aussi de sécuriser le transport.

Ainsi, la gestion des transports fut mise en place et se développa de manière cloisonnée, selon des visions stratégiques monomodales. Quelques passerelles subsistaient certes, mais leur généralisation dépendait autant d'une

24- Ainsi par exemple en 1931 l'État acheta et céda à la Compagnie de l'Elbe trois bateaux express pour la ligne Prague-Hambourg.

25- Ces considérations se limitent à la navigation à vapeur. Il y avait en $1930211 \mathrm{~km}$ de voies navigables en Bohème (Štěchovice-Prague-Mělník-frontière allemande) et environ 200 km en Slovaquie (la portion tchécoslovaque du Danube sur $172 \mathrm{~km}$ et du Váh entre Komárno et Guta sur $26 \mathrm{~km}$ ). Cependant, on pratiquait beaucoup la flottaison de bois aussi bien en Bohème (sur la Vltava ; Lužnice ; Otava ; Sázava), Moravie (Bečva ; Morava), Slovaquie (Morava ; Váh ; Hron) et Ukraine subcarpathique (Tisa et ses affluents). 
volonté politique plus affirmée que de progrès techniques qui les auraient rendues rentables. Le conflit rail-route, mais aussi l'évolution du contexte économique amenèrent de plus en plus d'observateurs à penser que la rationalisation de la gestion des transports devait nécessairement passer par la création d'un ministère commun.

\section{La genèse longue et difficile du ministère des Transports}

\section{La concurrence rail/route : frein ou moteur de la réforme?}

Nous avons vu que la jeune République avait à ses débuts tout simplement transposé les institutions autrichiennes et créa, par conséquent, un ministère des Chemins de fer en lieu et place d'un ministère des Transports, pourtant initialement envisagé2 ${ }^{26}$.

L'évolution de la conception de la gestion des transports que l'on put observer par la suite se fit selon trois phases successives très rapprochées dans le temps. Étant donné que seuls le transport ferroviaire et le transport routier se retrouvèrent dans une situation d'opposition, nous proposons, pour simplifier nos propos, de nous en tenir à ces deux modes de transport pour leur définition.

La première phase correspond à l'esprit du temps du "tout-ferroviaire " - une notion bien connue de l'historiographie française - hérité de l'ère austro-hongroise. La deuxième phase fut le moment où, la technologie du véhicule à moteur ayant fait ses preuves, il fut considéré comme un auxiliaire du rail dont l'emploi se limitait à des zones strictement définies (autour des gares; dans les agglomérations; dans les régions dépourvues de chemins de fer). Cette notion a dominé l'opinion dans les années 1920. Enfin, lors de la troisième, à partir du milieu des années 1930, le transport automobile se libéra de la tutelle ferroviaire et fut reconnu comme un mode de transport à part entière à la fois autonome et efficace.

Si nous voulions introduire la notion de concurrence dans le monde des transports tchécoslovaques, on ne pourrait considérer que le duel rail-route, car l'aviation et la voie d'eau furent toujours considérés comme ayant des caractéristiques intrinsèques complémentaires aux autres modes. Ce duel, nous pourrions le comparer à une petite " guerre des systèmes ", concept développé

26- En effet, le gouvernent provisoire envisageait le 2 novembre 1918 la création d'une « administration pour la gestion des transports » (úrad pro správu dopravy), dans l'esprit de tous le futur ministère des Transports. Cf. note 2. 
par Th. Hughes par rapport à l'industrie électrique ${ }^{27}$, mais applicable dans ses principes de façon plus globale.

La concurrence rail-route se fit sentir dès les premières années de la République. On a pu ainsi lire dans le Völkischer Beobachter en 1920 : « La concurrence des automobiles par rapport aux chemins de fer, considérée par l'administration ferroviaire il y a quelques années encore avec un sourire empreint d'un sentiment de supériorité comme une affaire dépourvue d'intérêt, commence à se développer au cours des derniers mois au point de devenir un danger sérieux pour le privilège du transport de marchandises par chemins de $\mathrm{fer}^{28}$. " Pourtant les ČSD prirent dès cette époque des mesures pour limiter ce qui fut compris rapidement comme « la concurrence» automobile. Leur poids économique et social et leur position politique les placèrent dans un rapport de force avantageux, ce qui ne manqua pas de peser sur le développement du secteur automobile privé (transport et industrie) à court et à moyen termes. Malgré quelques tentatives de réforme, le ministère resta l'organe regroupant les pouvoirs législatif, administratif, commercial, exécutif et de contrôle. Ce cumul de compétences semble avoir été néfaste à la construction d'un réseau de transport multimodal à l'échelle nationale, les rapports mutuels entre les différents modes étant trop souvent régis par la loi du plus fort.

Afin de réagir dans le contexte d'une concurrence grandissante et protéger son domaine de prédilection, le ministère des Chemins de fer disposait de différents outils - des leviers de pouvoir - que nous résumons ici :

Mesures externes : pression et lobbying auprès du législateur, réglementation des concessions des transporteurs ${ }^{29}$ ayant entraîné la taxation du transport routier, la limitation du rayon d'action des routiers aux concessions " irrégulières », la limitation du nombre de concessions pour des transports réguliers et irréguliers, la modification du statut du transport interne aux grosses entreprises industrielles pour les obliger à les concéder (à partir de 1935), l'exemption de toutes ces obligations pour les ČSD et les Postes, le traitement des demandes de concessions pour des lignes de bus par le ministère des Chemins de fer, etc.

27- Voir HUGHES, 1993.

28- « Die Konkurenzierung der Eisenbahn durch das Automobil, die noch vor wenigen Jahren seitens der maßgebenden amtlichen Eisenbahnkreise mit lächelnder Überlegenheit als der Diskussion unwerte Angelegenheit behandelt wurde, beginnt sich in den letzten Monaten zu einer ernsten Gefahr für das Frachtentransportprivilegium der Eisenbahn zu entwickeln », Völkischer Beobachter, juillet 1920.

29- Cf. par exemple l'article « Autobusová doprava » [Transport par autocar], Lidové Noviny, 11 décembre 1920. 
Mesures internes : organisation par les ČSD de leur propre transport routier ; coopération imposée aux transporteurs privés, notamment pour drainer le trafic vers des gares; mesures tarifaires (baisses des tarifs, modification des classes de marchandises, forfaits), négociation d'accords avec les industriels et les associations d'agriculteurs pour obtenir l'exclusivité de transport en échange de baisses tarifaires; fusion des compétences en matière de transport routier de l'État dans un seul ministère, celui des Chemins de fer, à partir du $1^{\text {er }}$ janvier 1933, préparée dès 1932 par la création au sein du ministère d'une "Administration centrale du transport routier " (Ústrední správa automobilové dopravy) ; création de "Spedra " (18 février 1934) $)^{30}$; introduction massive de la motorisation du trafic de voyageurs ; expérimentation des " trains mixtes" (comprenant des voitures à voyageurs et des wagons de marchandises) ; introduction de toutes sortes de mesures novatrices (téléphone et dispatching; traction à moteur ; services annexes, par exemple touristiques, livraison à domicile ; etc.).

Ces mesures, dont certaines relèvent d'une réelle volonté de réforme ou de l'innovation technologique ou organisationnelle, permirent une modernisation progressive du ministère et préparèrent l'avènement du ministère des Transports $^{31}$. L'hypertrophie du ministère des Chemins de fer eut pendant des années incontestablement un effet retardateur sur le développement du transport routier. Dans un contexte de domination économique d'un seul mode, il était difficilement envisageable de créer un ministère des Transports tant cette création était crainte comme un nouveau moyen de contrôle et donc d'asservissement du transport routier. La gestion des transports tchécoslovaques se trouva ainsi dans une impasse d'où elle allait être tirée par le changement du contexte politique et économique européen.

\section{L'influence des contextes politique et économique}

Il est évidemment impossible de traiter la question des transports en l'isolant du contexte politique et économique de son temps. Les liens et les interactions entre la politique générale et la vie économique, d'une part, et la coordination et la gestion des transports, d'autre part, étaient nombreux et permanents (nous en avons mentionné plusieurs, par exemple le rôle

30- ŠTEMBERK, 2004, p. 186.

31- «La concurrence du transport routier avait certes causé la perte d'une partie du trafic, la diminution des recettes et la fermeture de certaines lignes déficitaires, mais elle a en même temps obligé les ČSD à se moderniser », JAKUBEC, 1991, p. 76. 
stratégique des réseaux, leur indépendance physique et tarifaire sur le plan international, le monopole d'État, etc.). La période de l'entre-deux-guerres fut cependant marquée par deux événements majeurs, sans comparaison aucune avec les rapports qu'entretenaient ordinairement les différents acteurs. Il s'agit premièrement des effets de la grande crise économique déclenchée le 24 octobre 1929 à New York et, deuxièmement, de la crise politique internationale, liée à la montée du nazisme en Europe à partir de l'accession d'Hitler au pouvoir en tant que Chancelier de l'Empire allemand le 30 janvier 1933.

La crise économique avait apporté un durcissement de la concurrence rail-route. Les déficits budgétaires du ministère des Chemins de fer atteignaient alors des records. Le lien de cause à effet entre la concurrence automobile et le déficit ferroviaire, abusivement créé et entretenu pendant une dizaine d'années, fut paradoxalement, au plus fort de la crise, remis en question. Ce fut sous l'effet cumulé de l'expérience tirée des efforts faits pour surmonter la crise économique et de la montée d'un danger politique et militaire imminent que, progressivement, une nouvelle politique de transport commença à se mettre en place entre 1932 et 1935 . Voici ses lignes maitresses :

- réunir au sein d'un seul ministère le transport ferroviaire et routier public (1932);

- réformer les entreprises d'État en séparant les domaines de l'exploitation et de l'administration d'un côté et de la législation et de la supervision de l'autre ; appliquer dans l'entreprise publique le principe de la gestion commerciale (par exemple les réformes du ministre Hůla en 1932);

- adapter le service aux réalités du terrain, accélérer le processus d'application des innovations technologiques, comme par exemple la traction à moteur dans le domaine des chemins de fer ;

- reconsidérer le potentiel de chaque mode de transport à la lumière des réalités économiques et politiques nouvelles; développer l'intermodalité.

Ce dernier point retiendra notre attention, puisqu'il représente une volte-face partielle de la politique publique de transport. En effet, conscient de l'urgence et de la complexité de la situation, le gouvernement avait décidé de créer en avril 1935 la Commission pour l'élaboration d'un plan de transport global $^{32}$. Sa composition était interministérielle. Sa démarche pourrait se résumer ainsi : "La Commission part du point de vue que les transports sur terre, sur l'eau et dans l'air doivent être considérés comme un tout et

32- Komise pro sdèláni všeobecnébo dopravního plánu. 
chaque question les concernant regardée globalement ${ }^{33}$. " Les travaux de la commission allèrent dans un premier temps à l'établissement de statistiques, en particulier dans le secteur de transport routier privé. On s'aperçut qu'en fait on disposait depuis l'époque de l'empire austro-hongrois d'excellentes statistiques ferroviaires, mais que l'on basait les appréciations du transport routier sur des données incomplètes et des estimations souvent très floues ${ }^{34}$. Cependant, le travail de fond remarquable de la commission ne put porter tous ses fruits, car l'évolution de la situation politique extérieure devait précipiter les choses. Dès 1936, la construction d'une autoroute à l'exemple de l'Allemagne fut envisagée et, sous la pression du ministère de la Défense, le développement des véhicules à moteur à nouveau favorisé. Pour des raisons stratégiques, le véhicule à moteur fut placé sur un pied d'égalité avec le train. Dans les deux années qui précédèrent le démantèlement de la République, une importante campagne de sensibilisation de l'opinion publique en faveur de la motorisation vint compenser des années de négligence.

Le Traité de Munich priva en 1938 la Tchécoslovaquie d'une grande partie de son territoire. En même temps il anéantit vingt années d'efforts dans le but de construire un réseau ferré cohérent. La crise qui suivit le traité démontra clairement les insuffisances dans la gestion des transports et notamment les retards accumulés dans le domaine du transport routier. Elle précipita - et c'est tout un symbole - la création d'un ministère des Transports unique. Le 11 novembre 1938 fut la date de l'union des ministères des Chemins de fer et des PTT en un ministère des Transports. On annonça à cette occasion l'intégration du transport sur l'eau et aérien pour le $1^{\text {er }}$ juin 1939 et la sépara-

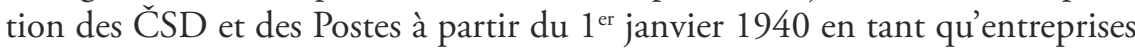
publiques distinctes du ministère à proprement parler (séparation des compétences réglementaires et économiques). Dans le gouvernement Beran ${ }^{35}$ le ministre des Transports fut un général de l'armée tchécoslovaque, le garant symbolique de la rationalité de la gestion.

33- Article du Ceské Slovo du 9 décembre 1935.

34- La Commission se chargea par exemple de créer une carte de transports de la ČSR ; d'établir un registre de poids lourds classés selon le tonnage et l'âge du véhicule ; un registre de toutes les entreprises de transport (bus et poids lourds), etc.

35- Le gouvernement Beran fut au pouvoir du $1^{\text {er }}$ décembre 1938 au 15 mars 1939. Il assura l'essentiel de la politique de la Deuxième République et mit en œuvre toute une série de réformes notamment dans le domaine des transports. Le ministre des Transports fut le général Alois Eliáš. 


\section{Conclusion}

La prise en considération de l'ensemble des modes de transports devait nous permettre de comprendre les enjeux et de mettre en lumière les solutions originales expérimentées dans ce domaine par la Tchécoslovaquie. Il s’agit là d'une esquisse de la " préhistoire " d'une approche multimodale des transports, sur le plan administratif, politique et économique.

En effet, durant toute la période étudiée, le chemin de fer était encore de loin le principal moyen de transport et moteur de la vie économique et sociale du pays. Devenu rapidement un monopole d'État, il subit cependant une concurrence croissante de l'automobile et cela autant dans le domaine des transports privés que des transports publics ${ }^{36}$. Cette situation, où les notions de coopération et de concurrence se mêlèrent rapidement dans les discours autant que dans les faits, ne fut pas toujours bien vécue et amena régulièrement sous la pression de l'opinion publique et celle des acteurs de la vie économique - des situations de conflits dont le paroxysme fut atteint en 1932-1933 dans le contexte de la dépression économique mondiale. Ce ne fut qu'à partir de ce moment-là que l'on commença à reconsidérer le rôle et la place de chaque mode de transport en y ajoutant une perspective plus rationnelle, plus complexe et déjà clairement multimodale.

La trame de fond sur laquelle se tissait l'histoire des transports tchécoslovaques était constituée du besoin vital de ce petit pays de soigner l'ensemble de ses possibilités de communication pour des raisons autant économiques que stratégiques. Les décisions et les engagements pris dans les premières années de la République furent déterminants pour toute la période de l'entre-deuxguerres, alors que la configuration générale de l'économie et de la vie sociale devait être bouleversée par l'arrivée de la technologie automobile et les progrès réalisés dans l'aviation. Ces choix, opérés encore à partir d'une expérience que l'on pourrait presque qualifier "d'ancien régime », car datant de l'empire austro-hongrois, pesèrent dans bien des décisions et actes législatifs comme autant d'archaïsmes dont on réclamait pourtant en 1918 à cor et à cri vouloir se défaire. À titre d'exemple, les détracteurs de la voie d'eau, aux yeux desquels le rapport entre des dépenses pharaoniques et le retour sur l'investissement pour l'économie était totalement disproportionné, ne purent obtenir que très

36- Ainsi, comme on l'a dit, le transport routier public était divisé jusqu'en 1932 entre deux ministères, celui des Postes et celui des Chemins de fer. Le ministère des Chemins de fer lui-même s'efforça de minimiser les effets concurrentiels de son propre transport routier. Il le confinait géographiquement dans des zones non desservies par la voie ferrée d'une part, et lui attribuait, d'autre part, le rôle de concentration et de distribution du trafic à partir et vers les gares. 
tardivement, malgré des preuves à l'appui, l'abandon définitif du grand projet de canal reliant les trois fleuves tchécoslovaques Elbe-Odra-Danube ${ }^{37}$.

Des rapprochements " inter-modaux » eurent lieu entre l'automobile et l'avion, entre la voie d'eau et les chemins de fer, puis, dans une configuration toute différente, car sous la houlette du seul ministère des Chemins de fer, entre les chemins de fer et le transport routier. Ces coopérations pesaient peu cependant dans la balance économique et la pensée des décideurs demeurait empreinte d'un cadre administratif et de pensée monomodal. Ce fut enfin sous l'effet de la crise politique internationale que les responsables politiques et économiques purent envisager une révision partielle du schéma de transport dont le plan d'urgence, conçu immédiatement après les traités de Munich, fut l'expression la plus pure. Il entraîna la création d'un ministère des Transports géré par un militaire, qui devait par ailleurs tenir compte du fait qu'une partie non négligeable des infrastructures et du matériel était perdue pour le pays. Il débloqua de nouveaux projets d'infrastructures comme celui du chantier de la première autoroute tchécoslovaque qui prenait symboliquement le relais des grands chantiers ferroviaires des années 1920.

\section{Bibliographie}

FETJÖ, 1988 : Fejtö, François (1909-2008), Requiem pour un empire défunt. Histoire de la destruction de l'Autriche-Hongrie, Paris, Points, coll. "Essais »,

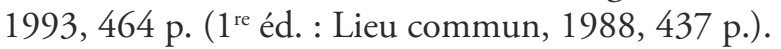

HUGHES, 1993 : Hughes, Thomas, Networks of Power, Electrification in Western Society, 1880-1930, Baltimore and London, The John Hopkins University Press, 1993 (Softshell Books Edition), 474 p.

JAKUBEC, 1991 : Jakubec, Ivan, Vývoj československých a nèmeckých drah 1929-1937 [Développement des chemins de fer allemands et tchécoslovaques 1929-1937], Prague, université Charles, 1991, 158 p.

ŠTEMBERK, 2004 : Štemberk, Jan, Podnikáni v automobilové dopravě $v$ českých zemich v první poloviné 20. století (Entreprendre dans le domaine des transports automobiles dans les pays tchèques de la première moitié du $20^{\mathrm{e}}$ siècle), thèse, Prague, 2004.

37- Voir par exemple F. Neubauer, employé des ČSD à Krásné Březno, « Naše vodní doprava na Labi a Vltavě, Její nepř́żnivý vliv národohospodářský » [Notre transport fluvial sur l'Elbe et la Vltava et ses influences néfastes sur l'économie], Želeænniční revue, n 3/1928. L'article analyse des exemples précis pour démontrer que la navigation intérieure fonctionnait certes à meilleur marché que le train, mais qu'elle ne participait pas au financement des aménagements très coûteux des voies navigables et que, somme toute, c'étaient les chemins de fer qui permettaient à la navigation d'exister en offrant des tarifs dégressifs sur de longues distances. 
